\title{
Les confréries et l'iconographie populaire des sept péchés capitaux*
}

\author{
JOANNE S. NORMAN
}

$D_{\text {ans les études médiévales, il est affaire courante d'interpréter les décrets }}$ du IVème Concile du Latran (1215) qui traitaient de la confession et de la pénitence comme des éléments catalyseurs de la pratique de prêcher plus fréquemment et plus régulièrement aux laïques. Mais l'ampleur des circonstances au niveau social et spirituel de cette nouvelle pratique ne fait que commencer à se faire connaitre. Le but premier de ces sermons était d'éveiller en la personne du pécheur une prise de conscience de son péché, de l'amener au repentir puis à la confession de ses fautes les plus graves. L'abondance régulière des sermons et les manuels pour faire les sermons qui en résultèrent ne diminuèrent pas pendant les siècles suivants. Bien au contraire, par la fin du XVème siècle, une demande quasi insatiable pour l'instruction et l'inspiration morales, appuyée par l'invention nouvelle de l'imprimerie, créa un véritable flot de matériels de ce genre. L'élément le plus frappant dans le développement de cette nouvelle spiritualité qui remonte au début du XIIIème siècle a été l'implication des laïques dans cette matière et l'influence qui en résulta. Chenu a défini ce mouvement, qui s'éloignait de l'expression monastique de l'expérience religieuse ascétique et détachée de ce monde pour aller vers un mouvement "qui encourageait la découverte des lois de la nature, formulait une prise de conscience de la Raison et de ses lois et déterminait la valeur des structures sociales," comme "un évangélisme apostolique." Cette nouvelle spiritualité trouva une de ces formes les plus appropriées dans les sociétés laïques organisées, les confréries et les guildes, dont la pratique chrétienne soulignait l'importance de la prière, de la pénitence, de la charité fraternelle et des principes moraux. Ce fut d'abord vers les congrégations urbaines des entrepreneurs que les ordres prêcheurs et mendiants orientèrent leur théologie sur la nature et la grâce, dans des sermons qui rejetèrent 
l'interprétation traditionnelle, allégorique ou figurée, des Ecritures, pour viser à un équilibre entre le sens propre et le sens spirituel. ${ }^{2}$ Le changement du contexte social pour les prêcheurs coïncida avec "une sensibilité aigue et croissante vis-à-vis des phénomènes naturels," un développement depuis longtemps reconnu dans le changement vers le naturalisme dans l'art du XIIIème siècle. La représentation réaliste de la faune et de la flore ainsi que des scènes de la vie quotidienne dans les sculptures des cathédrales se reflètent dans les sermons des XIIIème et XIVème siècles qui montrent un emploi plus fréquent d'exemples empruntés à l'histoire naturelle ${ }^{4}$ et aux occupations de l'homme. Une connotation morale était donnće à la vie séculière où l'éthique du comportement humain prennait une importance croissante et dont le ton devenait essenticllement pénitentiel. De plus, bien que l'alphabétisation se répandait au niveau de la nouvelle classe moyenne, les laïques continuaient de réclamer des images concrètes d’idées qui "could be conjured up in the mind with the help of a certain literary and spiritual tradition and also seen and lived over again by the fact that they were drawn, painted, represented for the eye to see in one way or another." C'est dans ce contexte de spiritualité laïque que nous devons voir la montée d'une nouvelle iconographie allégorique dans la décoration des églises paroissiales et des chapelles pénitentielles du XVème siècle. Cette iconographie présentait le conflit moral à l'intérieur même de l'âme humaine comme une procession des scpt péchés capitaux vers l'Enfer, chaque péché personnifié sous forme d'un homme ou d'une femme appartenant à une classe sociale bien distincte, chevauchant un animal symbolisant la nature bestiale et inhumaine de chaque vice.

La version la mieux connue de cette allégorie, tout au moins en littérature anglaise, est le défilé des sept péchés capitaux du Premier Livre du Faerie Queene de Spenser. Le caractère pictural des scènes de Spenser est frappant, et a naturellement mené les critiques modernes à rechercher, sans succès, une source directe pour cette partic de l'allégorie dans l'art contemporain. Les difficultés sont peut être apparues par le fait que cette procession des sept péchés capitaux ne fait pas partic de l'iconographic traditionnclle des vertus et des vices, mais est une nouveauté qui apparaît à la fin du XVème siècle et dont les origines restent quelque peu obscures.

L'allégorie visuelle la plus ancienne et la micux connue des Vices et des Vertus est celle d'une bataille entre des guerriers représentant les pulsions du bien et du mal dans la psyché humainc. Le concept provient à l'origine de la Psychomachia de Prudentius, une allćgorie du Vème siècle, et des 
illustrations qui en accompagnent le texte. L'iconographie de base, qui était chargée de connotations apocalyptiques dès le XIIème siècle et toujours associée aux églises de pélerinages et des monastères en général, montre une gucrrière armée écrasant impitoyablement un grotesque démon se tordant de douleur à ses pieds. Avec le XIIIème siècle, la métaphore du combat armé avait largement été remplacée par des représentations opposant vertus et vices, tel le fameux bas-relief de Notre Dame de Paris. ${ }^{6}$ Mais les vertus demeuraient toujours le centre d'intérêt de cet art. Comme le remarque Morton Bloomficld, "it is only in the later Middle Ages that the seven cardinal sins came into their own in painting and manuscript illustration."7 La nouvellc iconographic, qui avait recours aux péchés capitaux en tant que centre d'intérêt, ne venait pas d'une morbidité fin-de-siècle ni d'une fascination du mal, mais répondait plutôt à un intérêt croissant, en particulier chez les laïques, pour la conversion individuelle et le salut. Les confréries offraient les expressions visibles d'une piété renouvellée, basée sur le repentir et sur les pratiques spirituelles qui délivreraient des peines du Purgatoire:

C'est par les austérités que rivalisent orthodoxes et hérétiques, et c'est par elles que l'on espère fléchir Dieu, qui frappe si durement ses peuples. La pénitence est l'objet propre des premiers tiers-ordres; elle inspire toute une armée aux uniformes disparates; elle déchaîne la frénésie des flagellants de toutes bannières. ${ }^{8}$

Parmi les images du pénitentiel, la personnification des sept péchés capitaux tenait une place centrale. Leur influence en littérature et en théologie morale, en particulier dans les domaines pratiques du repentir et de la confession, a été amplement démontrée. Dans l'art, aucune allégorie morale des péchés ne remplacera la position dominante détenue par la psychomachia au XIIème siècle. Mais parmi la grande variété de représentations des Vertus et des Vices, la procession des sept péchés capitaux est particulièrement répandue en France. Une des particularités de l'iconographic plus tardive fut sa concentration en un seul endroit géographique. La psychomachia de l’art roman était véritablement d'ordre international et monastique, cependant dans la période tardive de l'art gothique, elle était française et séculière.

Un survol rapide des représentations de la procession des sept péchés pourrait aider à les replacer dans une perspective historique. Premièrement, il faut remarquer que tous les exemples, à l'exception d'un seul cas, présentent ce sujet sous forme de peintures murales, enluminures de 


\section{2 / Renaissance and Reformation}

manuscrits ou tapisseries. Souvent les études spécialisées traitent une oeuvre comme un phénomène local, créant par mégarde l'impression que ce sujet est particulier à une région de France. Bien qu'il semble que des exemples connus s'agglomèrent dans certaines régions, ils sont en fait dispersés sur l'ensemble du territoire français et font preuve d'une iconographie remarquablement constante.

La concentration géographique à l'intérieur même d'un seul pays se trouve aussi dans la définition chronologique. Bien que les deux exemples les plus anciens de la procession des péchés pourraient remonter à la fin du XIVème siècle, la plupart d'entre elles ont été réalisécs approximativement entre 1450 et 1520 . Le sujet appartient de manière très définitive au XVème siècle, et la procession des péchés peut donc être considérée comme une expression particulière de l'époque.

L'exemple le plus ancien connu jusqu'à présent a été peint à la fin du XIVème siècle dans la petite église 'paroissiale de St-Sulpice à Roussines (Indre), dans la vallée de la Creuse. ${ }^{9}$ (Fig. 1) Bien que les sept péchés capitaux soient distribués séparemment sur chacun des huit segments d'une voûte de la nef, l'idée d'une série est transmise par la disposition circulaire qu'impose la structure voûtée, par un arrière-plan commun, ainsi que par l'orientation des figures vers une scule et même direction. Comme il n'y a que sept péchés et huit segments à la nef, un moine en prière, les mains ligotées en signe de pénitence est peint sur la section supplémentaire. A l'origine, une banderole avec inscription identifiait chaque péché représenté. La banderole du moine n'est aujourd'hui plus visible; cependant, d'anciennes sources bien documentées assurent que figurait le mot "angustiae," faisant probablement référence à l'avertissement du prêcheur concernant les difficultés dans lesquelles se trouveraient ceux qui commettraient ces péchés.

Dans le sens contraire des aiguilles d'une montre, le premier péché est présenté sous forme d'un jeune scigneur à cheval, portant un faucon au poignet. L'habillement soigneusement recherché ainsi que le cheval justifient l'étiquette, orgueilh (Orgueil). A côté, l'Avarice tient une escarcelle à longue lanière et une coupe en or remplie de pièces de monnaies. Elle (sous des traits masculins). monte un quadrupède aux formes quelque peu ambiguës, pouvant ressembler à un ours. Le troisième péché, la Luxure, est présentée sous les traits d'un homme, se parade avec des attributs sexuels proéminants. Il tient une épée à la verticale et chevauche une chèvre. La Gourmandise apparaît sous la forme d'un homme gras, tenant une coupe 
bien remplie et un gigot, et se promène sur les dos d'un renard. La Colère, montée sur un porc, se poignarde. La Paresse, un mendiant aux pieds nus, suit à dos d'âne. Enfin, l'Envie est présentée par un marchand sur un lévrier rongeant un os. D'une main il tient sa bourse; l'autre est levée, en signe de défence.

Comme le montrent des exemples plus tardifs, les figures de Roussines ne présentent pas le modèle-type qui se développera par la suite. Pourtant, outre le fait d'être l'exemple pictural le plus primitif, ces figures montrent un nombre de caractéristiques significatives. Premièrement, elles représentent très distinctement les sept péchés capitaux, et non une série de vices indifférenciés face aux vertus. Ce sont des figures humaines, non des démons, et leur nature est revélée à travers des actes types de péchés particuliers ainsi que par l'animal symbolique qu'ils montent. Le soin donné à l'habillement contemporain aide à personnifier les péchés, tandis que les différences sociales, que reflètent aussi les costumes, suggèrent que chaque classe a ses propres faiblesses.

L'idée que certaines classes représentent des péchés individuels se montre encore plus évidente dans quelques enluminures de manuscrits français de 1392, qui sont en même temps un exemple primitif d'une série des sept péchés capitaux. ${ }^{10}$ Dans le B.N. ms. fr. 400 , chaque péché est représenté par un homme ou une femme montant un animal et tenant un oiseau symbolique. (Fig. 2) La plupart des péchés sont répartis à travers les différentes classes sociales: l'Orgueil, un roi sur un lion, tient un aigle; l'Envie, un moine sur un chien, tient un épervier; la Colère, une femme sur un sanglier, tient un coq; la Paresse, un paysan à dos d'âne, tient un hibou; l'Avarice, un marchand sur un blaireau, tient une corneille et une escarcelle; la Gourmandise, ceinte d'une épée et montant un loup, tient un cerf-volant; et, enfin, la Luxure, une femme sur une chèvre et tenant une colombe. Deux péchés, l'Envie et l'Avarice, font des gestes caractéristiques qui peuvent être comparés à ceux des péchés personnifiés à Roussines, mais la ressemblance la plus importante entre les illustrations du manuscrit et les peintures murales est la représentation très nette des sept péchés capitaux en hommes et en femmes d'époque caractérisant leur classe sociale. L'autre détail significatif est l'utilisation d'animaux pour suggérer la nature du péché personnifié par les figures humaines. Ces personnifications du péché et les animaux symboliques qui les accompagnent quelles chevauchent sont devenues l'iconographie de base de la procession des sept péchés capitaux. 
94 / Renaissance and Reformation

FIGURE 1: St-Sulpice, Roussines (Indre)

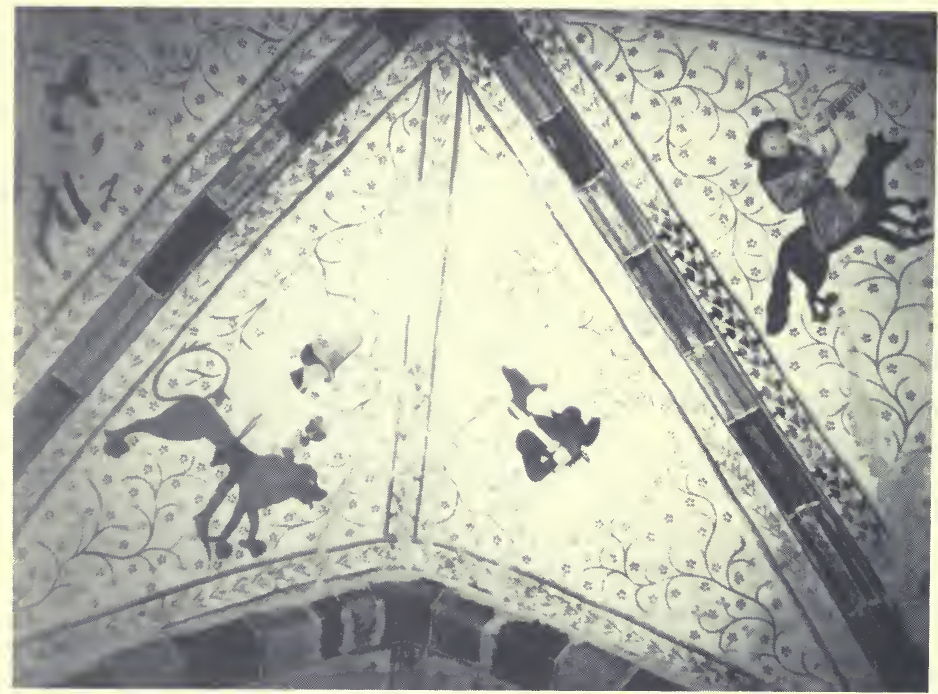

FIGURE 2: B.N. ms. fr. 400 f. 53

Photo. Bibliothèque Nationale, Paris

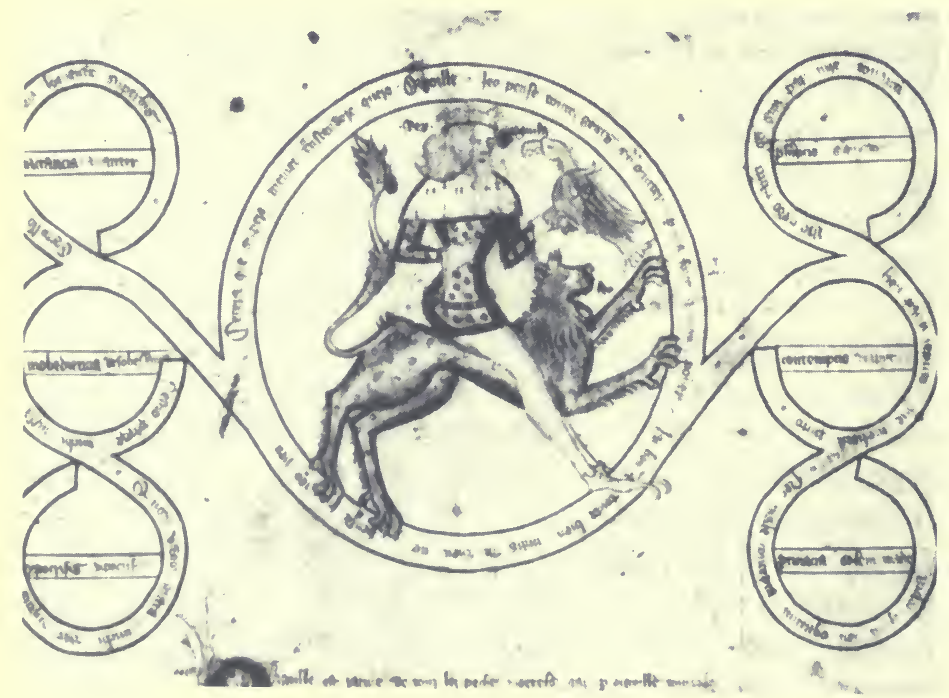


Les dessins du manuscrit sont encadrés d'une série de cercles entrelacés qui contiennent des phrases en français et en latin. Le texte français se conjugue à la première personne, par laquelle le péché s’identific; le texte latin, également conjugué à la première personne, ajoute une séric de péchés secondaircs relićs au péché principal. Chaque image a une légende en français décrivant les éléments-clé de l'ensemble; ces éléments sont aussi libellés en latin. Le texte que ces dessins doivent illustrer est une analyse hautement abstraite et systématique de chaque péché capital et de sa vertu réparatrice qui ne contient aucune allégorie ou ni même d'exemple concret. Les images servent d'aide-mémoire visuel mais leur symbolisme aurait été recueilli d'une autre source. Le ton d'exhortation ainsi que l'agencement de textes français et latins indiquent que cette forme de communication a pu servir, à l'origine, comme une aide pratique pour préparer un sermon orienté vers un public laïque et pour lequel l'emploi de la première personne donnait un sens de repentir.

Bien que les figures agissent en tant que symboles indépendants pouvant servir d'appui aux prêches, le sens qu'elles renferment n'est en aucun cas évident en soi. En fait, presque chaque fois que ces figures apparaissent dans l'art elles sont accompagnées d'une légende plus explicite. Cette nécessité d'interpréter les images est une reconnaissance formulée de la complexité et de l'ambiguité propres aux symboles, qui, au Moyen Age tardif, étaient détachés de tout texte explicatif. Elle a aussi des implications dans les contextes culturels et sociaux dans lesquels ces ocuvres étaient produites.

La représentation la plus ćlaboréc du thème de la procession des péchés fut réaliséc dans le sud-cst de la France, dans les régions alpines de la Savoie, du Piedmont et des Hautes-Alpes. Dans la vieille cathćdrale de Notre-Dame-du-Bourg, à Digne (Haute Provence), ${ }^{11}$ se trouve l'un des exemples les plus anciens (circa 1480) et les plus complets d'une peinture disposée en trois registres, typique de cette région. (Fig. 3) Du côté sud de la nef, le mur est décoré, tout d'abord, d'un Jugement dernier, suivi d'une vue de la Sainte Jérusalem; puis, à droite, d'une séric des sept vertus, des sept vices et des sept peines d'Enfer. (La première figure de chaque série est aujourd'hui effacée.) Dans le registre du milieu, les péchés sont présentés comme des individus chevauchant des bêtes symboliques, une lourde chaîne rattachant chacun à son compagnon. Ils apparaissent dans l'ordre suivant: l'Orgueil (aujourd'hui cffacé), l'Avarice, avec unc bourse, à dos de singe; la Luxure, une femme s'admirant au miroir, à cheval sur une truie; 
la Colère, un jeune homme se poignardant, sur le dos d'un lćopard; l'Envie, montant un lévricr, se montre l'oeil du doigt et regarde de travers ses voisins. La Gourmandise brandissant une cruche de vin, chevauche un renard, tandis que la Paresse, en haillons, se promène à dos d'âne et ferme la marche. Le registre supérieur renferme les symboles, tous féminins, des vertus. Assises ou agenouillées, elles exécutent des gestes contraires aux vices, qui leurs font face. Le registre inféricur présente les peines de l'Enfer assignées aux pécheurs. La composition picturale permet une lecture horizontale, de gauche à droite: vertus, péchés, punitions, et une lecture verticale: la vertu réparatrice, le péché mortel, et la punition du mal. (Fig. 4) La sélection des vertus et des punitions est fonction des péchés capitaux et souligne le fait qu'ils forment le centre de la composition. Le rôle-clé joué par les péchés capitaux est prouvé par l'existence de plusieurs peintures murales dans lesquelles seuls les péchés sont présents, alors que les vertus ou les peines apparantées n'apparaissent jamais dans l'absolu. Les peintures de Notre-Dame-du-Bourg sont accompagnées par d'assez longues inscriptions en latin et en provençal. Celles rattachées aux péchés capitaux sont à la première personne en langue vulgaire. Elles donnent le nom latin du péché, expliquent sa nature véritable et identifient son animal symbolique. Ces inscriptions permettent de faire un parallèle impressionnant au point de vue du fond et de la forme avec des inscriptions du BN. ms. fr. 400, exécutées cent ans auparavent et provenant du nord de la France.

Cette même iconographie, vue à Digne, devint la forme conventionnelle dans tous les autres exemples du sud-est, bien que les trois registres ne furent pas toujours présents. ${ }^{12}$ Ces peintures ne se trouvaient habituellement pas dans les grandes cathédrales mais dans les petites églises paroissiales ou même encore dans les petites chapelles. Les peintures étaient faites soit à l'intérieur, sur les parois de la nef, ou sur un mur extérieur protégé par les avancées du toit. (Fig. 6)

C'est donc à Les Vigneaux (1470), ${ }^{13}$ sur le mur extérieur du choeur de l'église paroissiale de St-Laurent qu'apparaît une peinture abîmée de la procession des péchés capitaux dans laquelle les vices humains, le cou enchaîné, défilent de la gauche à la droite: l'Orgueil, un jeune roi couronné, tenant un sceptre, monte un lion; l'Avarice, un marchand tenant unc boitte d'argent sur les genoux, monte un singe; la Luxure, chevauchant une chèvre, est vêtue à la dernière mode avec hermine et décolleté, lève coquettement sa robe, laissant paraître la jambe; l'Envie, les bras croisés, pointe du doigt dans des sens opposés et monte un lévrier qui ronge un os. La Colère, 
FIGURE 3: Notre-Dame-du-Bourg, Digne (Haute-Provence)

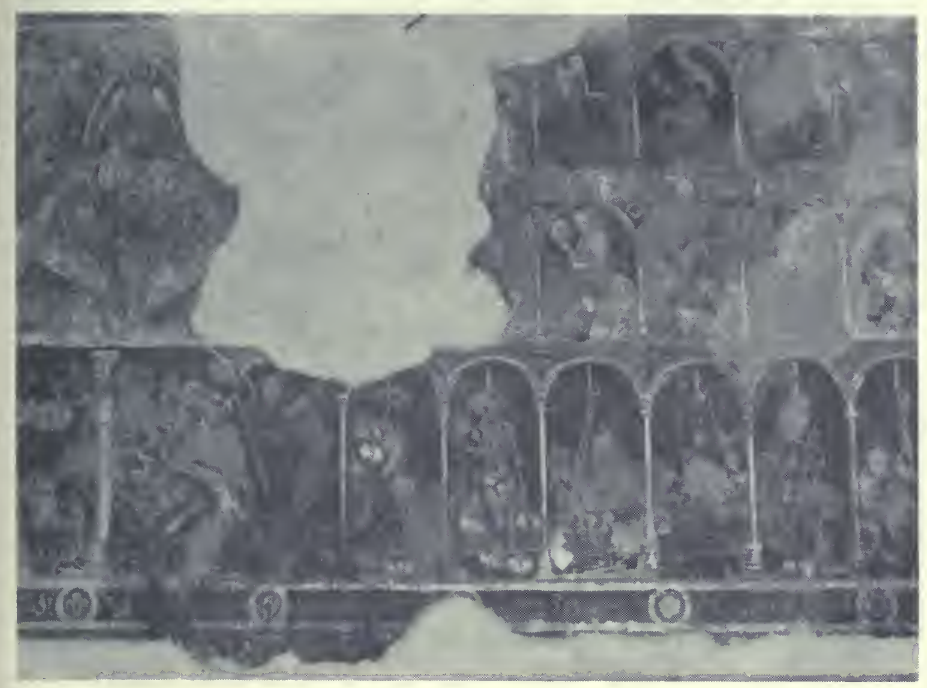

FIGURE 4: Notre-Dame-du-Bourg, Digne (Haute-Provence)

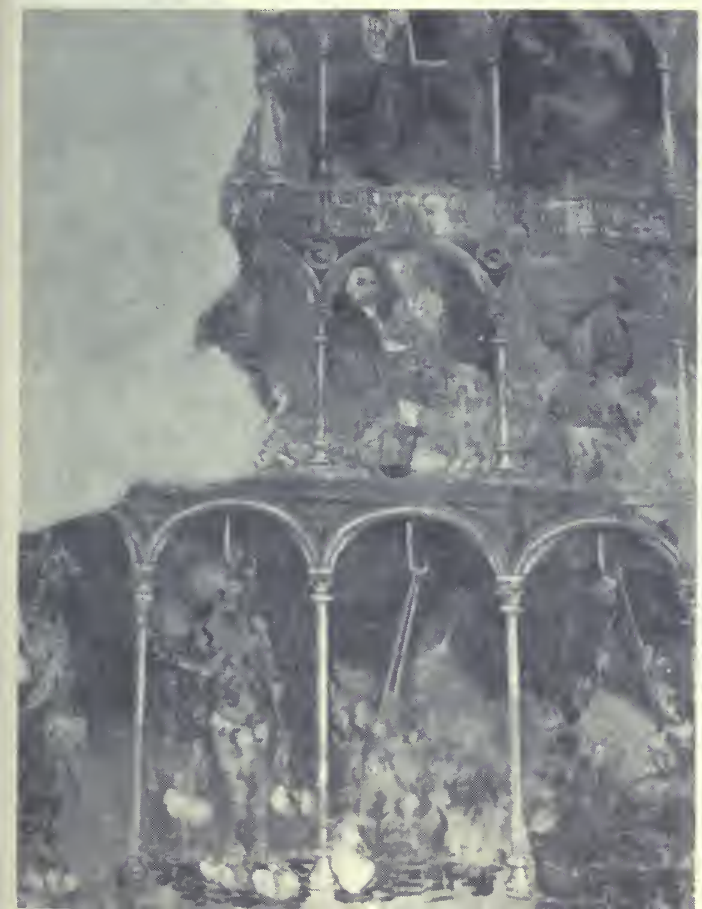


montant un léopard, se poignarde; la Gourmandise est un homme gras, buvant d'une coupe et brandissant un jambon, à dos de renard. La Paresse, en guenilles et aux cheveux ébouriffés, court aux côtes d'un cheval efflanqué. Tout à fait à droite, une immense bouche de l'Enfer, garnie de dents aiguisées, reste béante pour y recevoir les Péchés.

Sur le mur ouest de la Chapelle de Notre-Dame-des-Graces (à l'origine dédiée à St-Sćbastien, c. 1490)14 du petit hameau de Plampinet, se trouve un autre excmple d'une séquence à trois registres, typique de la région. (Fig. 5) Menés par le diable, les péchés, enchaînés, s'acheminent de gauche à droite vers les mâchoires de l'Enfer. L'Orgucil, monté sur un lion, tient comme d'habitude la première place; l'Avarice le suit, montée sur un sanglier. Vient ensuite la Gourmandise, sur un loup, la Luxure sur une chèvre, la Colère sur un léopard, l'Envic sur un lévrier et la Paresse, une femme sur le dos d'un âne. Des séries semblables, remontant au début du XVIème siècle, se trouvent dans la Chapelle St-Jacques à Prelles (c. 1450) ${ }^{15}$ et dans la paroisse de St-Appollinaire à L'Argentière-la-Besséc. ${ }^{16}$ (Fig. 6) Bien qu’il y ait de nombreuses variations de peu d'importance dans les détails, l'iconographie reste conforme à l'allćgorie du XIVème siècle. Les péchés sont représentés par différentes catégories humaines, mais les distinctions de classe et d'âge ont été estompécs. L’Orgucil apparaît parfois avec les symboles de royauté, parfois comme un fier noble ou bourgeois. La Paresse reste toujours un mendiant, homme ou femme. La Luxurc, contrairement à la figure masculine de Roussines, est presque toujours une femme de la haute société. Les autres péchés sont très peu différencićs et peuvent appartenir à la classe moyenne ou à l’aristocratic. Cependant, tous les péchés sont attribués à des laïques et seules certaines vertus sont attribuées à des religieuses.

Les péchés sont non seulement reliés par leur position relative mais aussi par la chaîne utilisćc pour exprimer l'idéc commune selon laquelle, au cours de la tentation humaine, un péché mène inévitablement à un autre. La monstrueuse bouche de l'Enfer, ainsi que le démon-guide, ont été ajouté afin de montrer sans équivoque le destin de la procession.

Les gestes symboliques des Péchés, les objets qu'ils portent ainsi que leurs montures respectives sont représentées de manière plus significative au XVème siècle. L'Orgueil est toujours vêtu somptueusement et porte souvent une couronne, un sceptre ou une ćpéc. Son animal est immanquablement un lion. L'Avarice a une bourse à la ceinture et tient souvent une boîte d'argent ou bien des sacs d'argent. Il monte un singe, un ours ou un sanglier. Les quatre autres péchés ne sont pas présentés dans un ordre conséquant. 
Renaissance et Réforme / 99

FIGURE 5: Notre-Dame-des-Graces, Plampinet (Hautes-Alpes)

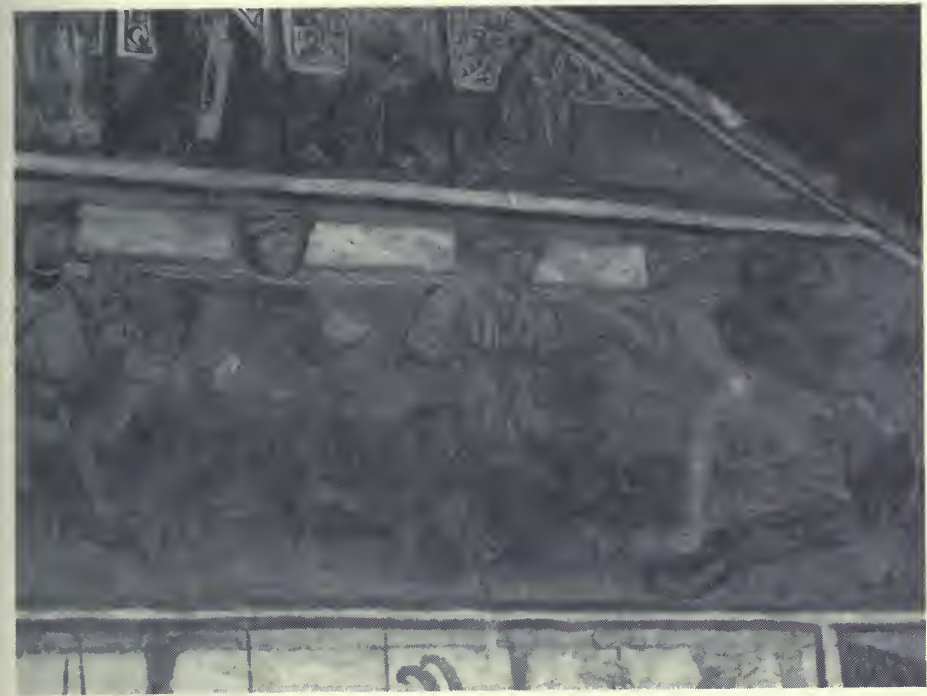

FIGURE 6: St-Appollinaire, L’Argentière-la-Bessée (Hautes-Alpes)

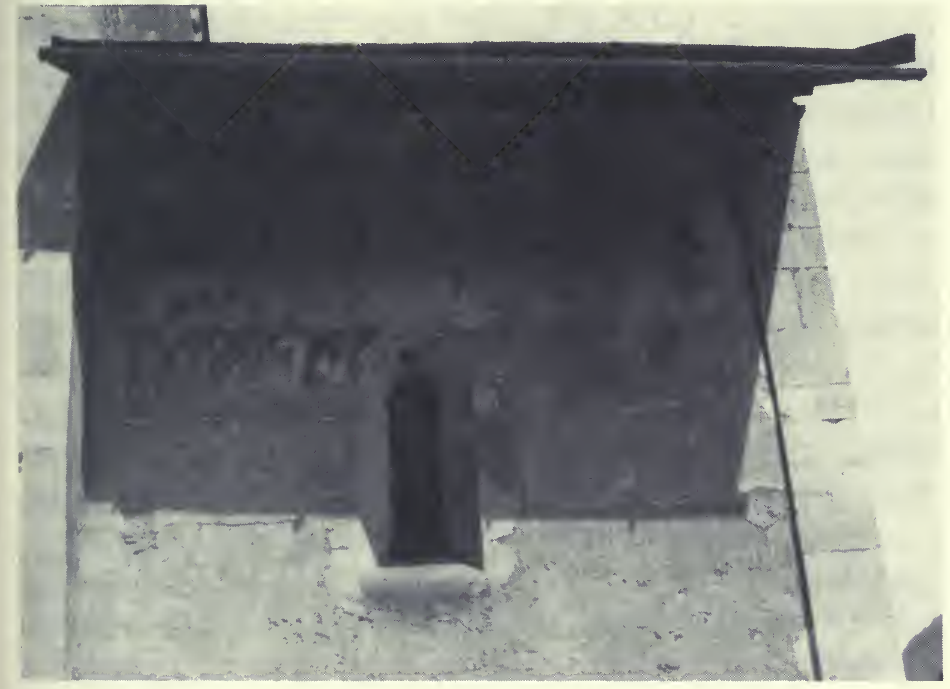


La Luxure, avec une robe décolletée, tient un miroir et lève ses jupes. Elle monte une truie ou une chèvre. La Gourmandise est toujours "gras," il boit d'un verre à vin ou d'un flacon, et tient en brochette un jambon ou un poulet. Son animal est le renard, ou lc loup. La Colère en montant un léopard se poignarde un sujet qui remonte à la représentation de l'Ira par Prudentius. L'Envie monte un lévrier, et se croise les bras pour pointer dans deux sens contraires. Ce geste particulier peut être interprété comme un signe extérieur du désir pervers de l'hypocrisic, la nature essentiellement contradictoire du péché, puisque cette attitude se jetrouve régulièrement dans l'art roman pour représenter le mensonge et la contradiction. Enfin, la Paresse, en guenilles, ferme la marche, à dos d'âne.

Douze peintures murales traitant ce même sujet ont ćté trouvées dans les régions alpines de la Savoie et du Piedmont, provenant toutes d'églises de villages situés à l'entour du passage du Mont Cenis. Ces villages semblent isolés, dans les montagnes, mais les montagnes donnent accès à l'Europe toute entière et le passage du Mont Cenis, plus particulièrement, reliait l'Italic au cocur de la France. Les pélerins et les marchands empruntaient les grandes routes de montagnes pour aller vers l'Allemagne du Sud, la vallée du Rhin, l'Italic, la France, les Pays-Bas, et même l'Angleterre. ${ }^{17} \mathrm{Le}$ long de ces mêmes routes de montagnes, la région de chaque côtés de la frontière franco-italienne, le plus souvent sous domination française jusqu'au XVIIİ̀me siècle, formait une entité culturelle où les influences françaises et italiennes étaient également importantes dans l'art régional. ${ }^{18}$

Le même mélange de styles se trouve plus au sud, où les peintures murales des chapelles des Alpes-Maritimes présentent la procession des sept péchés capitaux. Ces exemples existent dans les régions rurales qui, encore aujourd'hui, sont pauvres, isolćes et difficiles d'accès. L'apparition des premières manifestations de la peste, à la fin du XVème siècle, mena, en dépit de difficultés quasi-insurmontables, à la construction de nombreuses chapelles rurales le long des chemins menant aux villages des alentours de Nice. Ces chapelles étaient habitucllement situécs à quelque distance même du village. ${ }^{19}$ Elles étaient dédiées aux saints populaires et semblent presque avoir été construits selon le même plan. ${ }^{20} \mathrm{La}$ chapelle typique a une seule nef rectangulaire avec une porte dans le mur ouest et surmontée par une fenêtre. (Fig. 7) Sur la partie inféricure des murs de côté apparaissent les Vertus et les sept Péchés capitaux, tandis que les autres peintures représentent habitucllement la vie d'un saint ou des scènes de la Passion. Les Vertus et les Péchés accompagnent souvent une scène du Jugement 
Dernier mais ils peuvent aussi apparaître seuls, ce qui rappelle l'iconographie de la Savoie-Piedmont. Mais, le triple registre de peintures venant du Nord se transforme en une composition plus simple formée de deux bandes parallèles, une de Vertues féminines debout, l'autre d'une procession à dos d'animaux des sept Péchés capitaux, habituellement situées de chaque côté de la nef.

La chapelle de St-Sébastien à Roubion, ${ }^{21}$ construite en 1513, peut être considérée comme un proto-type de l'iconographie de cette région. Sur le mur nord se trouve une procession de Péchés, menée par l'Orgueil en jeune seigneur chevauchant un lion jusqu'en Enfer. Suivent l'Avarice, un vieux marchand tenant un sac d'argent dans chaque main, à dos d'un animal ressemblant à un chien; la Luxure, avec son miroir, à cheval sur un chamois; la Colère, qui se poignarde deux fois, monte en amazone un dragon ailé; la Gourmandise qui boit d'un flacon (Fig. 8a) et tient un rôti en brochette, est à cheval sur un sanglier; l'Envie, sur un renard, montre du doigt son "mauvais" oeil et pose la main sur son poignard; la Paresse est un mendiant endormi sur le dos d’ânc, dont les brides traînent. Ces Péchés, comme ceux des Hautes-Alpes, portent une chaîne autour du cou. Un démon cornu tire le bout de cette chaîne par dessus l'épaule, entraînant le groupe dans la bouche de l'Enfer tandis qu'un autre démon-squelette joue du pipeau et du tambour afin d'accélérer le défilé. (Fig. 8b)

Le démon musicien est un détail particulier à Roubion qui suggère une "danse" des Péchés. En effet, un des Péchés à Digne fait allusion à la procession comme à une "danza." Un parallèle pourrait être établi, comme cela a déjà été suggéré, entre la "danse macabre" et la procession des Péchés. Pourtant, aucune "danse des péchés" n'existe en peinture et le sujet a été uniquement traité dans un poème ćcossais d'époque, Dance of the Sevin Deidly Synnis de William Dunbar qui reflète plusieurs thèmes propres au gothique tardif. 22

Le style habituel de la peinture de Roubion et des chapelles environnantes, ainsi que des détails importants au niveau de la symbolique, telles que les figures du diable et de l'Envie, distinguent ces peintures et démontrent qu'elles ne sont pas des imitations directes des processions venant plus au nord à la même époque. Il y a un contraste stylistique frappant au niveau de l'élégance et de la vivacité des figures de Plampinet, dont les peintures révèlent le style italien bien marqué, exécuté par un artiste très doué, ${ }^{23}$ et le style de Roubion qui a été décrit comme "rustique et naif" 24 ou encore "populaire et puéril." 25 Pourtant, le concept de base est le même. 
102 / Renaissance and Reformation

FIGURE 7: St-Sébastien, Roubion (Alpes-Maritimes)

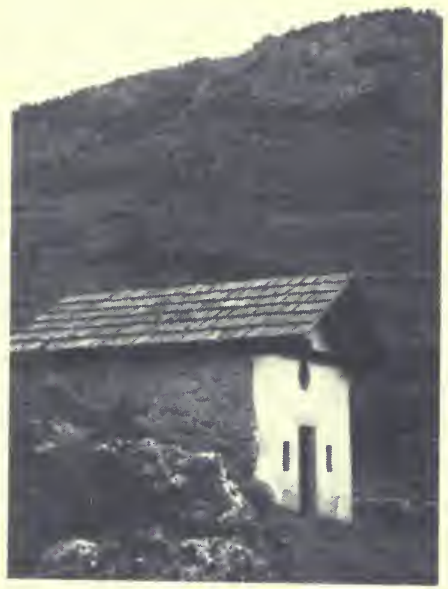

FIGURE 8: St-Sćbastien, Roubion (Alpes-Maritimes)

a. Colère et Gloutonnie

b. Démon et Orgeuil
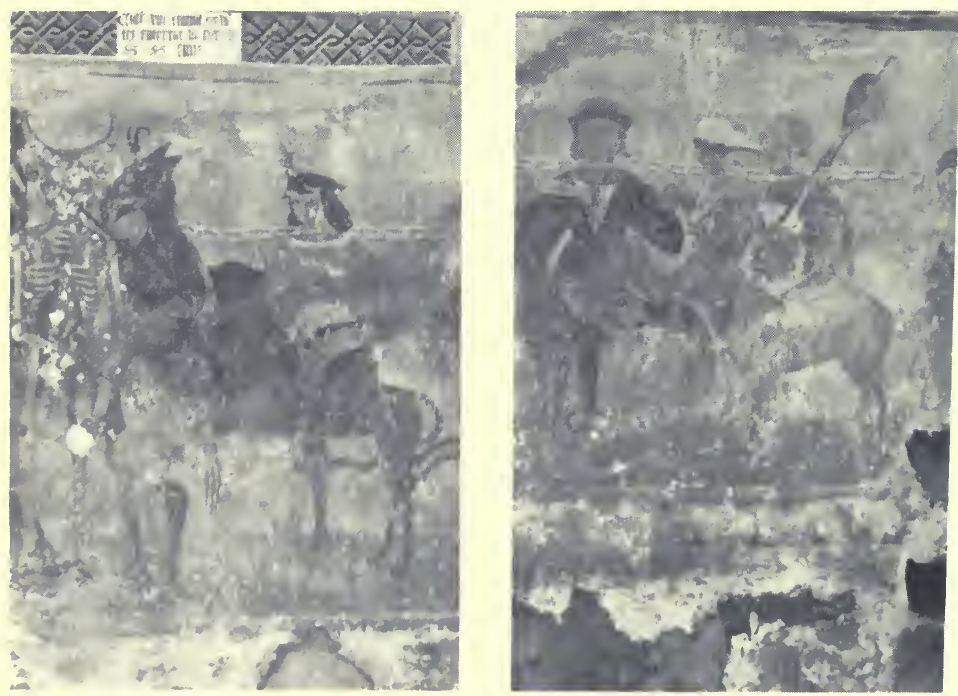
La clé pour comprendre le dévcloppement du sujet de la procession des sept péchés capitaux se trouve non pas dans l'analyse stylistique d'exemples individuels, mais dans l'appréciation des raisons pour lesquelles ils étaient peints et de leurs sources communes. Puisque l'évidence documentée est fatalement incomplète, pcu d'assertions générales peuvent être avancées avec certitude, mais celles-ci ont des sous-entendus plus larges. La procession des sept péchés capitaux s'est développée dans des régions qui étaient habituellement défavorisées, isolées des grands centres du pouvoir du mécenat de l'aristocratie. Ceci dit, ces régions étaient situées sur les routes de commerce, ce qui facilitait la communication et l'échange d'idées entre les peuples. Lorsqu'un tableau a été attribué à un peintre celui-ci semble généralement être soit de la région-tel serait le cas du peintre de Plampinet, dont la descendance pourrait encore y vivre, ${ }^{26}$ soit de l'étranger-tels les artistes de Digne qui ont manifestement été formés dans le nord de la France, ${ }^{27}$ ou encore venant de la Lombardie ou du Piedmont, comme ceux de l'Argentière. ${ }^{28}$ La situation devient plus complexe lorsque l'on constate que la peinture de Plampinet a de proches affinités avec la procession des Péchés de Villafranca, de style italien, peinte par un artiste français d'Oulx..$^{29}$ La décoration des chapelles niçoises était réalisée par des groupes d'artistes itinérants de la région et de l'étranger, qui se spécialisaient en peinture murale et qui semblaient ouverts au même mélange d'influences françaises et italiennes que les peintres de la Savoie. ${ }^{30}$

Dans le sud de la France, l'art des villages était limité aux églises locales et encouragé par des mécènes de la région ou, plus souvent, par la communauté toute entière. Roubion, par excmple, avait été construit par Jean et Erige Lubonis de Clans, de la famille du prêtre local, ${ }^{31}$ tandis qu'une chapelle avoisinante était "fieri communitas venansoni." 32 Les fresques dans la chapelle des Pénitents, à La Tour, étaient peintes "per magistros curraudi brevesi et guirardi nadali pittores de nicia et compatres in nomine domini." 33 Le fait que toutes ces chapelles furent bâties dans une période de temps relativement courte et à des fins analogues, explique la similitude de leur plan architectural, relativement simple, et de leur iconographie, essentiellement conservatrice.

Les peintures des sept Péchés capitaux semblent jusqu'ici ĉtre concentrées dans le sud-est de la France: les détails varient largement à partir de l'exemple plus tôt de Roussines. Une autre série de peintures, cette fois dans le sud-ouest de la France, surtout dans la vallée du Lot et la province de Guyenne, montre pourtant que le concept n'était par limité aux Alpes. 
Les peintures les plus connues de ces régions, qui ont été reproduites dans le Palais Chaillot à la suite d'une méticulcuse retauration, sont celles de la petite paroisse de l'église de St-Pierre-c̀s-Liens, à Martignac. ${ }^{34}$ Toutes ces peintures semblent dater du tout début du XVIc̀me sic̀cle, et traitent le même sujet. A Martignac, une série de Vertus avec une procession des sept Péchés capitaux occupent tout le mur nord de la nef. Les péchés sont représentés comme des personnes aisées, chacunes montant un animal symbolique et toutes enchaînées l'une à l'autre par la taille. (Fig. 9) Au lieu d'avoir un seul démon debout à la bouche de l'Enfer, ici, chaque péché a son propre démon bien identifié, tel astarot ou bescabuc. Le talent artistique du peintre de Martignac lui a permis de donner un caractère individuel à chacun des cavaliers et de montrer la motivation psychologique qui sous-entend les péchés. Il est malheureux que la détérioration des peintures empêche de reconnaître le détail avec certitude.

Dans une église du village de Pervillac (Fig. 10), du même diocèse, il existe une peinture d'une procession de Péchés qui remonte probablement plus loin que celles de Martignac mais dont la conception est semblable. ${ }^{35}$ C'est encore tout le mur nord de la nef qui est peint, rempli ici par une série de Vertus, indifférenciées debout et des sept péchés capitaux. Chaque figure est soigneusement étiquettée. Chaque péché est accompagné par son démon qui le mène sur le chemin de la facilité et tous les péchés sont enchaînés l'un à l'autre par la taille. Le premier est l'Orgueil, un jeune noble portant un chapeau avec une plume et une chaîne en or. Il tient un sceptre et chevauche un lion. La Luxure, une femme coiffée à la mode du jour, vêtue d'une robe décolletée, monte une chèvre et tient un miroir. La Paresse, à la robe déboutonnée, suit à dos d'âne. La Gourmandise est un goinfre corpulant qui tient un jambon. Il est monté sur un sanglicr. L'Avarice, à cheval sur un ours ou un loup, tient ses sacs d'argent, tandis que l'Envie, qui pourrait être un moine, se croise les mains pour pointer dans les sens opposés. Sa monture est malheureusement presque entic̀rcment effacée, mais on pourrait deviner un dragon. La Colère, qui ferme la marche, est sur un léopard, et se poignarde. Face aux Vertus et aux Pćchés du mur nord se trouve la Sainte Jérusalem, et les tourments de l'Enfer sont peints sur le mur sud.

Un autre exemple de la procession de Péchćs dans la même région se trouve en l'Eglise de la Masse, à Les Junics. ${ }^{36}$ Ici, l'Orgucil est non seulement coiffé d'un chapeau à plumes et à cheval sur un lion, mais il tient aussi un faucon au poignet, ce qui rappelle Roussines. L'Avarice, avec sa bourse et 
Renaissance et Réforme / 105

FIGURE 9: St-Pierre-ès-Liens, Martignac (Lot)

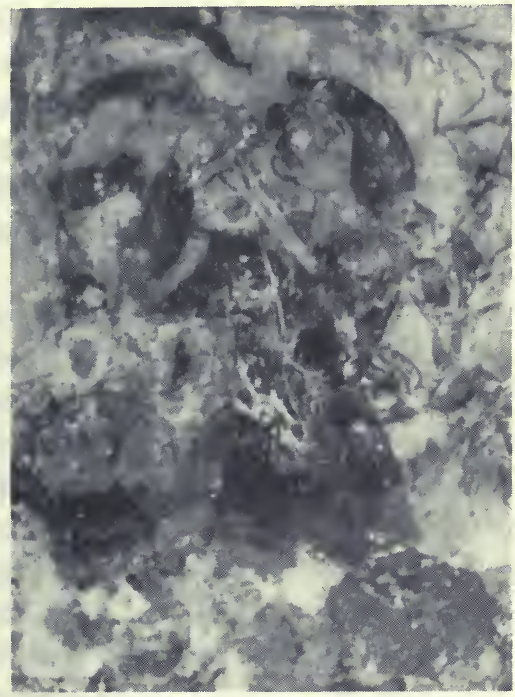

FIGURE 10: Pervillac (Tarn-et-Garonne)

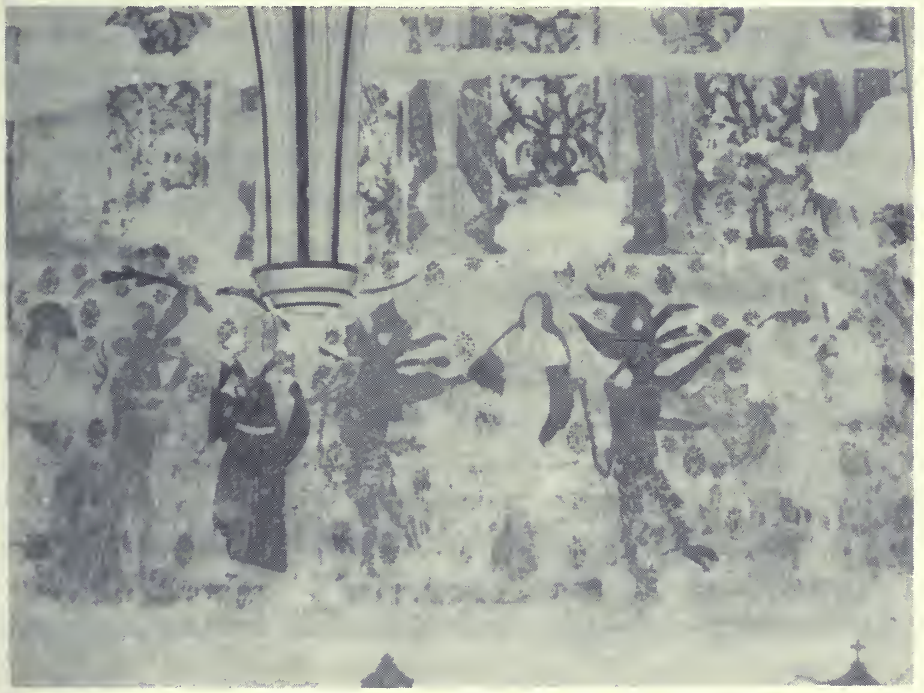


ses sacs d'argent, monte aussi un ours, tandis que la Luxure, la Gourmandise, la Colère et la Paresse restent sur leur animal respectif. D'autre part, l'Envie pointe en direction de son oeil et se tient l'estomac, toujours monté sur un lévrier. Cette représentation de l'Envic réunit des éléments de Roubion et de Plampinet. Dans cette peinture, ce sont les animaux, plutôt que les cavaliers, qui sont enchaînés et le diable les tire jusqu'en Enfer à l'aide d'une chaîne passée par-dessus son épaule, et chaque péché est aussi accompagné par un diable.

Les affinités de ces processions de Péchés avec celles du sud-est de la France sont évidentes. Ces exemples sont moins systématiques dans la représentation des détails. Aussi, l'ordre des Pćchés est bousculé et certaines bêtes symboliques sont soit changécs, soit données à d'autres cavaliers. L'innovation la plus frappante est le remplacement du diable qui mène la procession par des diables individuels qui accompagnent chaque péché dans la bouche de l'Enfer. De tels excmples se trouvent dans la région du Piedmont, dans la chapelle St-Etienńc à Jaillons et dans celle des Horres. ${ }^{37}$ Dans cette dernière, datée de 1506, le diable tire les cheveux de la Luxure, aide la Colère à se poignarder et malmène la Paresse. A Jaillons chaque Péché est tourmenté par un démon, l'Avarice monte un ours et la Paresse est une femme à moitié vêtue: ces deux détails rappelent Pervillac.

La présence de diables individucls donne une connotation différente à l'interprétation des figures représentant les Péchés. Lorsqu'elles sont seules, elles peuvent être interprétées comme étant le Pćché abstrait soit personnifié, soit incarné. Sa forme humaine représente le niveau terrestre ou mortel où le Péché agit; cette interprétation de la position du péché est renforcée par sa place occupée dans le registre central de la représentation tripartite. L'apparance extérieure du Péché doit demeurer quelque peu ambiguë, ce qui donne à son animal symbolique un signe visible de sa véritable nature intérieure. L'Avarice n'est plus simplement un homme avare mais l'idée même de l'avarice est concrétisée. La présence de démons individuels brouille cette distinction clairc. Si les diables agissent comme temptateurs, comme à Pervillac et à Martignac, ou comme les tourmenteurs de Jaillons, la figure humaine devient plus victime que sujet agissant. Le diable se charge de motiver le Péché tandis que le cavalicr est réduit à celui qui commet l'acte repréhensiblc. Les compagnons du démon ne sont pas toujours présents dans d'autres exemples de la procession des sept péchés capitaux trouvés dans le sud-ouest de la France. Dans l'église de St-Martin à Champniers (Vienne), une procession, où les cavaliers ne sont pas 
accompagnés, apparaît une fois de plus comme scène secondaire à un Jugement Dernier. ${ }^{38}$ Les images de l'Enfer recouvrent la totalité du mur de l'ouest et il n'y a pas de figures des Vertus.

Une autre église dédiée à St Martin à Pommeraie-sur-Sèvre (Vendée) ${ }^{39}$ a aussi une scène de procession sans Vertus ni scène du Jugement Dernier. Les Péchés sont enchaînés par la taille et un diable à cornes les tire vers la gigantesque bouche de l'Enfer. (Figs. 11a et 11b) Une fois de plus, les Péchés sont habillés à la mode de la haute société de l'époque de Louis XII ou du début du règne de François I. On note des changements intéressants pour chacun des Péchés. L'Orgueil est un jeune roi sur un lion. L'Envie porte un vêtement bordé d'hermine et monte une panthère. D'une main il montre ce qu'il désire tandis que l'autre se tend pour l'attrapper. L'Avarice est un vicux marchand avec une bourse à la ceinture qui monte un ours. La Luxure est exceptionnellement un jeune homme sur une chèvre, qui rappelle les figures de Roussines. Le gros Gourmand tient une tasse et gruge une cuisse de poulet, son porc mange aussi, dans une auge. La Colère porte une armure et monte un griffon. Il a une expression sauvage et se transperce le corps de son épée. Fermant la marche, la Paresse, pieds nus, est endormie sur un âne. Un diable bleu la fait avancer à coups de gourdin. Aucun de ces péchés n'est nommé, mais un soin tout particulier a été pris pour rendre leur action très explicite. Il semble y avoir à nouveau un intérêt à établir une correspondance entre les péchés et les classes sociales et d'en attribuer une grande partie à la classe des nobles. Le péché semblerait être relativement profitable dans ce monde, sinon dans l'autre, car dans la procession les Péchés poursuivent leur route plus ou moins sains et saufs.

Bien qu'il ne soit pas possible ici de discuter tous exemples des sept péchés capitaux, une autre procession mérite un bref aperçu en tant qu'exemple tardif, provenant d'une région éloignée de notre centre d'étude. Une modeste structure du XVème sic̀cle, l'église Notre-Dame, du village de Bourisp, dans la vallée de l'Aure (Hautes Pyrénées) fut peinte en 1592.40 Dans le porche se trouve une série complète de dessins des sept péchés capitaux, tous chevauchant les animaux symboliques, maintenant familiers. Les péchés sont représentés par des dames vêtues avec élégance, derrière lesquelles un diable ailé parle à l'oreille.

Les peintures murales françaises de la procession des sept Péchés capitaux posent un nombre de questions intéressantes concernant la nature de ce sujet dans l'art et le contexte dans lequel il se trouve. En premier lieu, il y a le problème de la localisation des ocuvres. Aujourd'hui, on connaît 
un seul exemple de la procession des péchés en art en dehors de la France. Il s'agit d'un linteau sculpté de la chapelle Roslin en Ecosse, probablement fait par des artistes français. En termes historiques, les églises du Piedmont et de la Lombardie qui contiennent ces peintures faisaient partie de la France. Il est évident que le concept des sept Péchés capitaux était connu de façon universelle à travers l'Europe, et les illustrations de manuscripts des péchés chevauchant des animaux symboliques étaient représentés dans d'autres pays, principalement l'Allemagne, l'Autriche et les Pays-Bas. Mais les peintures murales ou les sculptures représentant les sept péchés capitaux dans d'autres pays se refèrent à une iconographie toute autre. Outre les limites géographiques, presque toutes les peintures connues ont été réalisées entre 1470 et 1592 . L'iconographie de la procession enchaînée de figures humaines sur des animaux reste un phénomène particulièrement français de la fin du XVème siècle.

Il reste donc à savoir quelles circonstances particulières auraient pu engendrer le développement de ce thème. Les peintures faisaient toujours partie d'une église ou d'une chapelle paroissiale locale, commandées par des individus laïques, tels des mécènes de la région ou des artisans bien établis, ou par la communauté elle-même. Les chapelles niçoises se révèlent ici particulièrement intéressantes puisqu'elles montrent la grande influence des confréries de pénitents laïques dans la subvention, la construction et la décoration de ces édifices. ${ }^{41}$ La conscience sociale et l'encouragement d'une vie chrétienne dans ce monde, pour une récompense juste dans le prochain, que les processions de péchés exemplifiaient, ont du être très compatibles avec la moralité praticante et la piété laïque que les confréries devaient développer. A l'origine, ces sociétés étaient associées avec les ordres monastiques, il est donc naturel qu'elles soient apparues dans des régions où l'influence monastique s'était faite sentir. ${ }^{42}$ Ceci était vrai, bien sûr, pour les régions alpines où les Chartreux et les Augustiniens avaient été particulièrement dominant. Le XVème siècle vit les confréries au sommet de leur popularité et de leur influence, surtout en France et en Italic. ${ }^{43} \mathrm{~A}$ cette époque, elles étaient devenues plus au moins des organisations autonomes, non plus sous le contrôle monastique mais essentiellement associées aux divers métiers dans les villes et les villages.

Cette audience n'était pas particulièrement sophistiquée en matière d'art, mais elle était relativement cultivée et possédait un bon entendenent des doctrines de base de la foi. Le rôle central joué par les sept péchés capitaux dans l'enseignement de la confession et de la pénitence expliquerait leur 
importance dans les peintures laïques dans des chapelles pénitentielles et des églises paroisiales. C'est pour les laïques éduqués que ces figures étaient soigneusement identifiées et que des commentaires écrits étaient ajoutés. ${ }^{44}$ Leur fonction première était d'enseigner et d'inspirer une attitude adéquate envers les choses spirituelles. L'image était une façon saisissante, voire dramatique, et rendait l'apprentissage aisé et servait d'aide mémoire. Une fois qu'un thème particulier avait été adopté par une confrérie, cette association participant au mouvement continu du commerce, contribuait à propager les thèmes d'une région à une autre.

Bien que les confréries peuvent aider à expliquer l'apparition des processions des sept Péchés capitaux dans des régions éloignées l'une de l'autre, elles ne forment pas une source véritable. En effet, l'iconographie pouvait aussi trouver ses sources dans les représentations théâtrales locales. Les inscriptions à Digne, par exemple, suggèrent une dramatisation de base. Les confréries sont bien connues pour leur contribution significatives au théâtre de la fin du Moyen Age et la plupart des manifestations publiques semblent avoit inclues des processions. Cependant, une ou deux pièces de théâtre de Savoie qui nous sont parvenues contiennent des dramatisations des sept péchés capitaux, qui ne sont pas nécessairement des processions symboliques. En fait, les pièces étaient de date postérieures aux peintures et donc la question de "qui influence qui" demeure sans réponse. Roques conclut que "Il y a pour les uns [peintures] et les autres [mystères] une source commune que nous ne connaissons pas encore." 45

Une autre source possible qui serait encore plus familière à un public laïque serait le sermon. Le travail de Richard et Mary Rouse et de Siegfried Wenzel a montré l'importance croissante à travers le Moyen Age du sermon en langue vulgaire, plus particulièrement pour l'enseignement moral et les appels au repentir. Il existe plusieurs sermons analysant les Péchés de chaque classes sociales ou groupes; quelques uns attribuent plus d'un Péchés à une même classe. ${ }^{46}$ Une grande partie des sermons puisent intensément dans le thème des sept Péchés capitaux traditionnels. Les confréries, à travers leur insistance sur la fréquentation de la messe et des offices liturgiques et pénitentielles, auraient souvent exposées leurs membres à ce type de sermon. Un manuel de prêcheur en particulier, l'Etymachia, présentait les sept péchés capitaux chevauchant des animaux symboliques. Ce manuel était devenu très populaire dans le sud de l'Ạllemagne, la vallée du Rhin et les Flandres pendant la seconde moitié du XVème siècle. ${ }^{47}$ Il a été souvent reproduit sous forme de manuscrits 
110 / Renaissance and Reformation
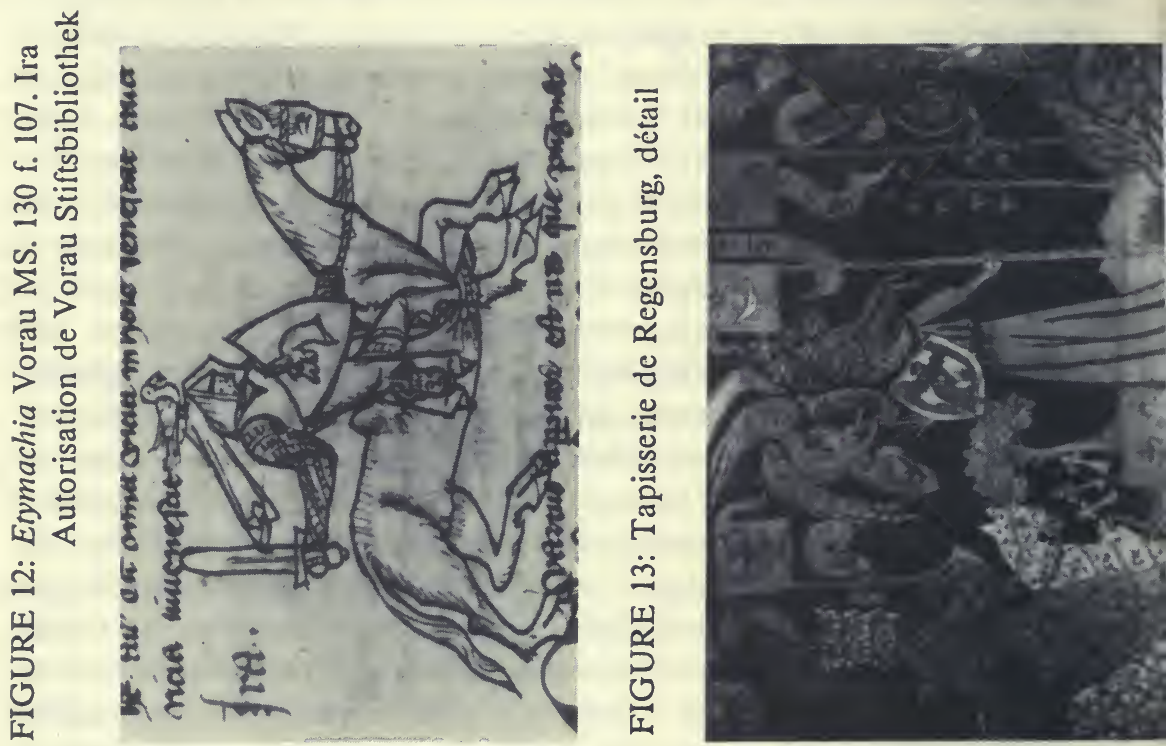

ป
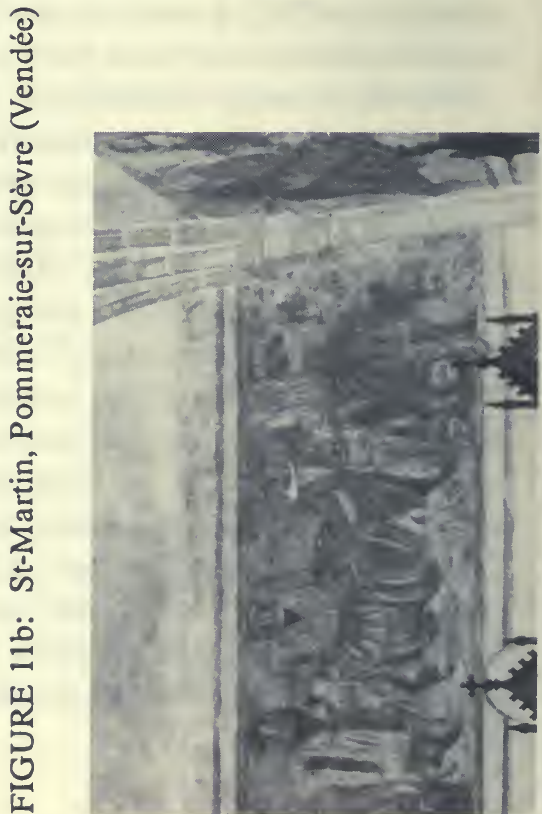
illustrés ainsi que dans des éditions imprimées en langue vulgaire. (Fig. 12) Les illustrations se sont petit à petit détachées du texte afin de paraître tels des dessins indépendants dans d'autres manuscrits, gravures sur bois et tapisseries allemandes ou flamandes du XVème siècle, comme par exemple la très ancienne tapisserie de Regensburg. (Fig. 13) Un manuscrit allemand de Würzburg, daté entre 1412 et 1426 , contient des instructions détaillées pour une peinture murale qui viennent directement de l'Etymachia, mais qui incorporent des éléments, tels que la procession en file de figures humaines montées sur des bêtes symboliques, une bouche de l'Enfer et une escorte de diable, qui rappelle beaucoup les peintures murales françaises. ${ }^{48}$ Emile Mâle, qui présenta l'étude la plus complète de cette iconographie, a dû reconnaître l'influence potenticlle du traité Etymachia lorsqu'il identifia le B.N. ms. fr. 400 comme ćtant l'Etymachia bien que ni le texte ni les images n'appartiennent réellement au traité. Ces dessins de manuscrits, pourtant, comme les peintures murales, présentent les sept péchés capitaux chevauchant des animaux symboliques d'une façon qui ressemble à l'allégorie de l'Etymachia. Ne serait-il pas possible pour les confréries françaises, à travers l'emploi commun du matéricl de l'Etymachia dans les sermons et à travers les relations commerciales avec les Flandres et l'Allemagne, d'absorber cette nouvelle allégorie visuelle des scpt péchés capitaux et de l'utiliser comme thème dans leur art religicux local?

Mais, quelque soit son origine précise, la procession des sept péchés capitaux reflète bien les intérêts intellectuels courants et les préoccupations religieuses de la bourgeoisie française, dont l'indépendance et l'influence croissaient, à la fin du Moyen Age.

\section{Université d'Ottawa}

\section{Notes}

* Traduit de l'anglais par Marie-Louise Higham et Marguerite Higham.

1 M.D. Chenu, Nature, Man, and Society in the Twelfih Century, traduit par Jerome Taylor et Lester K. Little (Chicago: University of Chicago Press, 1968), pp. 202-38.

2 Gabriel Le Bras, "Les confréries chrétiennes: problèmes et propositions," Revue historique de droit français et étranger (1941-42): 348-49 et Chenu, pp. 251-62.

3 Chenu, p. 232.

4 Mary A. et Richard H. Rouse. "The Texts Called Lumen Anime," Archivum Fratum Praedicatorum 41 (1971): 15.

5 Jean Leclerq, "Abstract and Popular Spirituality in the Late Middle Ages," (dissertation présentée au Congrès international sur le Moyen Age, à Kalamazoo, MI., 1984). 


\section{2 / Renaissance and Reformation}

6 L’étude générale de cette évolution dans l'art du Moyen Age a été faite par Adolf Katzenellenbogen, Allegories of the Virtues and Vices in Mediaeval Art from Early Christian Times to the Thirteenth Century, traduit par Alan J.P. Crick (1939; Leichtenstein: Kraus, 1968).

7 Morton W. Bloomfield, The Seven Deadly Sins (Ann Arbor: Michigan State University Press, 1952), p. 103.

8 Le Bras, p. 322.

9 Cette église n'est pas très connue. Il y a très peu de resources documentées disponibles. Quelques lignes ont été écrites par Jacques S. Sacy dans le Dictionnaire des églises de France, Belgique, Luxembourg, Suisse, vol. 4 (1966). Emile Mâle a aussi contribué par une brève description dans L'art religieux de la fin du Moyen Age en France, Sème édition révisée (Paris: Colin, 1949), p. 333.

10 Seul E. Mâle donne une description détaillée de ce manuscrit, p. 332.

11 Marguerite Roques, Les peintures murales du sud-est de la France: XIIIème au XVIème siècle (Paris: Picard, 1961), pp. 156-59; M.J. Roman. "Le tableau des Vertus et des Vices," Mémoires de la société des antiquaires de France 41 (1880-81): 29-30; Camille Blanchard, "L'art populaire dans le Briançonnais: les Vertus et les Vices," Bulletin de la société d'études historiques, scientifiques et littéraires des IIautes-Alpes (1923): 196 et Ollivier, "Dignes et ses environs," Anmales des Basses-Alpes et Bulletin de la société scientifique et littéraire des Basses-Alpes (1881-83): 38,76 et 131.

12 Les descriptions de toutes les églises sont basées sur les photos et les observations tirées de mes propres recherches. Un certain nombre de peintures parait dans la liste de la Commission des monuments historiques à Paris, dont les resources documentées ont toutes été examinées. Dans la plupart des cas il y a très peu de matériel. Toutes études régionales auxquelles j’ai pu avoir accès apparaît sous forme de notes en référence à chacunes des églises. A part ces notes de référence, les études suivantes offrent un aperçu plus général ayant trait aux régions dans lesquelles se trouvent les peintures murales: Lucien Bégule, Les peintures murales à Lanslevillard et Bessans (Lyon: Rey, 1918); Camille Blanchard, "L'art populaire dans le Briançonnais: les Vertus et les Vices," Bulletin de la société d'études historiques, scientifiques et littíraires des IIautes-Alpes (1921): 36-43, 114-28; (1922): 62-72, 180-204; (1923): 193-237 et G.Sentis, L'art du Briançonnais: la peinture au XVème siècle (Gap: Louis-Jean, 1970).

13 Roques, pp. 263-64.

14 Roques, pp. 48, 304-307; Blanchard (1921), p. 37; B. Faucher, "Un septième exemplaire dans les Hautes-Alpes de la peinture des Vertus et des Vices, découvert à Plampinet," Bulletin de la société d'études historiques, scientifiques et littéraires des IIautes-Alpes (1920): 29-33.

15 Roques, pp. 49, 55, 233; Blanchard (1922), pp. 159-65.

16 Roques, pp. 48, 385-87; Blanchard (1922). pp. 133-65.

17 J.E. Tyler, The Alpine Passes: The Middle Ages (962-1250) (Oxford: Blackwell, 1930), pp. 48-52, $143,162$.

18 R. Oursel, Art en Savoie (n.p.: Arthaud, 1975), pp. 17-19 et Roques, pp. 120-21.

19 Latouche, Robert, "Les peintures murales des chapelles de Saint Sébastien de Roubion, de Saint Sébastien de Roure et de la Madonne de la Roquette," Comptes rendus et mémoires ... de l'Institut historique de Provence (1927): 92.

20 Roques, pp. 15-16 et Paul Canestrier, "Les chapelles rurales et les saints populaires du comté de Nice," Nice historique (1946): 3-4, 10-12. 
21 Debidour, p. 20; Roques, pp. 50, 380-82.

22 La relation entre le poème de William Dunbar et les sujets de procession des sept péchés capitaux a fait l'objet d'une de mes études intitulée "Sources for the grotesque in William Dunbar's Dance of the Sevin Deidly Synnis," qui sera publiée dans Scottish Studies, 1988.

23 Roques, p. 307.

24 Victor-Henry Debidour, Trésors cachés du pays niçois (Paris: Hachette, 1961), p. 20.

25 Roques, p. 382.

26 Blanchard (1921) 37, note 4.

27 Ibid. (1923), p. 238.

28 Roques, p. 387.

29 Ibid., p. 307 et Blanchard (1923), p. 202.

30 Latouche, pp. 92-99.

31 L. Imbert, "Les chapelles peintes du pays niçois," Nice historique (1947): 16-18 et Latouche, pp. 93-94.

32 Debidour, pp. 125-26.

33 Ibid., p. 131.

34 Ici encore, il semble y avoir peu de resources documentées pour cette église et sa décoration. Il y a une brève note écrite par Marguerite Vidal dans le Dictionnaire des églises. D’autres descriptions sont données par Robert Mesuret dans Les peintures murales du sud-ouest de la France du XIème au XVIème siècle (Paris: Picard, 1967), p. 274; Yves Bonnefoy, Peintures murales de la France gothique (Paris: Hartmann, 1954), p. 32 et Bulletin monumental (1943-44): 147-48.

35 Mesuret, pp. 229-30; F. Pottier, Bulletin archaéologique et historique de la société de Tam-etGaronne, 20 (1892): 330-31 et M. Méras, "Les peintures de Pervillac," Bulletin de la société archaéologique de Tarn-et-Garonne (1963): 32-37.

36 Mesuret, p. 114.

37 Blanchard (1921), pp. 62-65 et (1923), pp. 180-84.

38 René Crozet, Dictionnaire des églises.

39 Ibid.

40 Raymond Rey, L'art gothique du midi de la France (Paris: Renouard, 1934), pp. 311-12 et Victor Allègre, Dictionnaire des églises.

41 Voir Canestrier, Debidour et Latouche. Par exemple, Latouche traduit la charte fondatrice de Roubion:

... en raison de la dévotion particulère que vous manifester pour le ... Christ Sauveur et pour Saint Sébastien par les prières et l'intercession justement de la peste et de beaucoup d'autre maladies diverses et qui obtient de nombreuses grâces... vous désirez... faire construire, élever et édifier une chapelle sous le vocable de Saint-Sébastien auprès et en dehors de l'enceinte du lieu de Roubion....(94)

42 Le Bras, pp. 314-15; Canestrier, pp. 3-4.

43 Joseph Duhr, “Confréries," Dictionnaire de spiritualité ascétique et mystique, vol. 2, 1470-80.

44 Roques, pp. 75-78.

45 Ibid., p. 47. 
46 Ruth Mohl, The Three Estates in Medieval and Renaissance Literature (New York: Ungar, 1962), p. 257.

47 L'unique recherche détaillée de ce traité fait partie intégrante d'une étude sur les manuscrits de Lumen animae par Richard et Mary Rouse. Les images ainsi que l'influence de l'iconographie des sept péchés capitaux ont été l'objet de ma dissertation intitulée "A Practical Illustrated Handbook for the Fifteenth-century Preacher."

48 Cette description a été fournie par une note dans le Pierpont Morgan MS. 298, une collection de plans détaillés de sermons et exempla faits pour son usage propre par Johannes Sintram de Herbipoli, un écrivain Franciscain, érudit et prêcheur de Wurzburg. Je suis reconnaissante envers le docteur Nigel Palmer, Oriel College. Oxford, pour m’avoir montrée ce manuscrit et autorisée à la lecture de sa transcription de cette note. 